\title{
MODULATION DOMAIN REFERENCE POINT DETECTION FOR FINGERPRINT RECOGNITION
}

\author{
Nantapol Kitiyanan and Joseph P. Havlicek \\ School of Electrical and Computer Engineering \\ University of Oklahoma, Norman, OK 73019-1023 USA \\ E-mail: yongie@ou.edu joebob@ou.edu
}

\begin{abstract}
Accurate reference point detection is one of the first and most important signal processing steps in automatic fingerprint identification systems. The fingerprint reference point, which is also known as the core point except in the case of arch type fingerprints, is defined as the location where the concave ridge curvature attains a maximum. In this paper we introduce a multi-resolution reference point detection algorithm that calculates the Poincare index in the modulation domain using an AM-FM model of the fingerprint image. We present experimental results where this new algorithm is tested against the FVC 2000 Database 2 and a second database from the University of Bologna. In both cases, we find that the modulation domain algorithm delivers accuracy and consistency that exceed those of a recent competing technique based on integration of sine components in two adjacent regions.
\end{abstract}

\section{Introduction}

Automatic fingerprint recognition continues to assume an increasingly important role in biometric identification and verification applications. Two prints of the same finger taken at different times and under different imaging conditions may be expected to differ by translation, rotation, elastic distortion, and noise; thus, a practical fingerprint recognition algorithm must tolerate all of these. Prior to recognition and matching, it is therefore advantageous to register the two fingerprints with respect to one another using some well-defined set of reference points that must be automatically extracted from the fingerprint images. In [1], the (single) reference point was defined as the point of maximum curvature in the fingerprint concave ridges. This definition coincides with the traditional core point for loop type fingerprints and with the upper core point for whorl and double loop type fingerprints. It also extends the notion of reference point to arch type fingerprints, which do not have a core point in the traditional sense.

The ridges and valleys in ideal fingerprint images tend to be highly coherent and characterized by slowly varying orientation and contrast. Thus fingerprints are ideally suited for AM-FM image modeling. The FM part of the model captures the local ridge orientation and inter-ridge distance, while the AM part captures the local contrast between ridges and valleys. In [2], AM-FM Dominant Component Analysis (DCA) was used to extract the dominant AM-FM component from fingerprint images in the NIST database [3,4]. By using the dominant AMFM image component instead of the NIST-enhanced fingerprint image for input to the standard NIST PCASYS classification algorithm, the authors obtained significantly reduced misclassification rates. AM-FM DCA was also used with a reaction-diffusion process in [5] to repair occlusions in fingerprint images.

In this paper, we focus on the problem of automatically detecting the reference point in fingerprint images using the definition of reference point that was proposed in [1]. We introduce a new multi-resolution algorithm that estimates the reference point by calculating the Poincare index in the modulation domain using the dominant AM-FM image component extracted by DCA.

\section{AM-FM Image Models}

AM-FM functions are nonstationary quasi-sinusoidal oscillations that admit simultaneous amplitude and frequency modulations [3]. Let $s(x, y)$ be a real-valued fingerprint image. Let $t(x, y)$ be the complex analytic image associated with $s(x, y)$ by adding an imaginary part using the 2-D directional Hitbert transform [6]. Then $t(x, y)$ can be modeled as a multi-component AM-FM function of the form $[3,4]$

$$
t(x, y)=\sum_{k=1}^{K} t_{k}(x, y)=\sum_{k=1}^{K} a_{k}(x, y) \exp \left[j \varphi_{k}(x, y)\right] .
$$

In (1), $a_{k}(x, y) \geq 0$ is the $\mathrm{AM}$ function of component $t_{k}(x, y)$, which describes local contrast. The FM function $\nabla \varphi_{k}(x, y)$ describes local texture orientation and granularity; it is related to the familiar horizontal and vertical frequency fields by $\nabla \varphi_{k}(x, y)=\left[U_{k}(x, y) V_{k}(x, y)\right]^{T}$.

At each pixel, our goal is to estimate the AM and FM functions of the component $t_{k}(x, y)$ that dominates the local image structure at the pixel. Collectively, these are known as the dominant image modulations. The estimates of the dominant FM function are also often referred to as the emergent frequencies of the image. We isolate the multiple image components in (1) from each other by applying a multi-band Gabor filterbank as described in [4]. By construction, we define the component-wise decomposition of the image given in (1) such that the response of each filterbank channel is dominated by exactly one image component. We have then that $y(x, y)$, the response of Gabor filter $G_{i}$, is dominated by some component $t_{k}(x, y)$ in (1). Thus, $y_{i}(x, y)$ is given by $y_{i}(x, y)$ $=t(x, y) * g_{i}(x, y) \approx t_{k}(x, y) * g_{i}(x, y)$, where $g_{i}$ is the filter impulse response. Estimates of $a_{k}(x, y)$ and $\nabla \varphi_{k}(x, y)$ for all components can be obtained from the filterbank channel responses using the local nonlinear demodulation algorithm [3] 


$$
\text { and } \quad \begin{aligned}
\nabla \varphi_{k}(x, y) & \approx \operatorname{Re}\left[\frac{\nabla y_{i}(x, y)}{j y_{i}(x, y)}\right\rfloor \\
a_{k}(x, y) & \left.\approx \mid \frac{y_{i}(x, y)}{G_{i}\left[\nabla \varphi_{k}(x, y)\right.}\right\rceil .
\end{aligned}
$$

To perform DCA, we must identify at each pixel the filterbank channel that contains the dominant AM-FM image component. We do this by selecting the channel that maximizes the selection criterion

$$
\Psi_{i}(x, y)=\frac{\left|y_{i}(x, y)\right|}{\max _{\omega_{1}, \omega_{2}} \mid G_{i}\left(\omega_{1}, \omega_{2)} \mid\right.}
$$

Use of this criterion was motivated in $[3,4]$. The dominant modulations are generally quite robust against noise and provide a rich, powerful description of the local fingerprint structure. The argument of the emergent frequency vector is normal to the local ridge orientation, while the reciprocal of its magnitude gives the inter-ridge distance. The dominant AM function describes the intensity differences between a ridge and the surrounding valleys. However, we have found that the AM information is generally not required to obtain satisfactory performance in detecting the reference point. Thus, the reference point algorithm we propose here makes uses only the dominant FM information.

\section{Filterbank Design and Segmentation}

For general AM-FM image analysis, one typically utilizes a bank of Gabor filters that densely samples the entire 2-D frequency plane. However, since fingerprint images tend to have well defined local frequencies and orientations that occur in relatively narrow bands, it is possible to greatly reduce the processing complexity required for DCA in our application by designing a Gabor filterbank that is specifically tuned for fingerprint images.

We have found that a 16-channel filterbank such as the one shown in Fig. 1(a) is sufficient to isolate image components, capture ridge structures, and remove unwanted background regions. The frequency origin is located in the center of Fig. 1(a) and the depicted quantity is $\left|G_{(}(u, v)\right|$ for $u, v \in[-0.5,+0.5]$. The positive horizontal frequency axis points to the right and the positive vertical frequency axis points down. Thus, quadrant I of the frequency plane is in the lower right sector of the figure, and a clockwise path beginning in quadrant I and encircling the origin traverses quadrants II, III, and IV. Note that the fiterbank covers only quadrants I and IV: This is because the Fourier spectrum of the analytic image $t(x, y)$ in (1) has no support in quadrants II and III [6]. A detail view is shown in Fig. 1(b), where filterbank channel numers are also superimposed.

The filterbank design depends on a parameter that tunes the outer ring of filters to the average inter-ridge distance for the database. Let $f$ be the average ridge frequency (reciprocal of the average inter-ridge distance) expressed in cycles per image (cpi). Then filters in the outer ring in Fig. 1 all have radial center frequency $f$, while filters in the inner ring have radial center frequency $f / 1.8$. While our reference point detection algorithm is reasonably insensitive to the parameter $f$, at least a coarse $a$ priori estimate of $f$ is neecled, e.g., to distinguish between a database containing images of single fingerprints and a database where each image contains prints of multiple fingers.

Each filter in Fig. 1 has a half-peak radial bandwidth of one octave and any four adjacent filters intersect at a single frequency where all four are precisely at half-peak. Each ring consists of eight filters with center frequency orientations spaced equally in the right half-plane. Filters in the outer ring (those with odd channel numbers in Fig.1 (b)) capture ridge structures in the fingerprint image, while those in the inner ring (even channel numbers in Fig. 1(b)) capture the background. Note that the exact angular spacing between filters is not critical: the FM algorithm (2) will estimate the dominant frequencies with a high resolution irrespective of the filter bandwidth. What is required is that the filter spacing must be sufficiently dense to reject cross-component interference between multiple AM-FM image components. In other word:, there must be enough filters to support an interpretation (1) of the image as a sum of $K$ locally coherent AM-FM components $t_{k}(x, y)$.

Our approach for obtaining an initial segmentation of the fingerprint from the background is exceedingly simple. For each pixel, if the dominant AM-FM component as defined by (4) is contained in a channel with an even number (inner ring of filters), then the pixel is classified as background. If instead the channel maximizing (4) has an odd number, then the pixel is classified as foreground (part of the fingerprint). This simple approach gives only a very rough, approximate segmentation. We then refine the binary map of region labels by first smoothing it with a morphological open-close filter and then applying connected components labeling with minor region removal to retain only the largest connected component - which will correspond to the fingerprint. This segmentation procedure is illustrated in Fig. 2. Fig. 2(a) shows an original fingerprint image from the FVC 2000 DB2 [7]. The initial segmentation is shown in Fig. 2(b). Fig. 2(c) shows the refined result after postprocessing. The final segmented fingerprint is shown in Fig. 2(d).

\section{Orientation Field Estimation}

In this section, we describe our algorithm for estimating the fingerprint local orientation field. This field is a smooth function that gives the local orientation of the ridges and is generally orthogonal to the dominant frequency field $\nabla \varphi_{D}(x, y)$. Our algorithm is based on the elegant approach given in $[9,10]$ for solving the orientation field from the gradient of the image. Two main ideas are involved. First, the granularity of $\nabla \varphi_{D}(x, y)$ is too fine: local spatial averaging is needed to estimate the desired smooth orientation field. Second, the orientation field generally comprises unsigned angles $0 \leq \theta \leq \pi$. Thus, two adjacent frequency vectors represented as complex numbers $r_{1}=e^{j \theta}$ and $r_{2}=e^{j(0+\pi)}$ should result in an averaged orientation not of zero, but of $\theta \bmod \pi$.

Following $[9,10]$, we achieve this by spatially averaging the squared dominant frequency vectors. The effects of squaring are twofold. First, the frequency magnitudes are squared, but that is of no consequence here, since we are only interested in estimating orientation. Second, the angles of the dominant frequency vectors are doubled, which eliminates the problem of 
opposing vectors averaging to zero orientation. With regards to the example above, we obtain for the average of the squared vectors $1 / 2 r_{1}^{2} r_{2}^{2} e^{j(20+2 \pi)}$, which has orientation $2 \theta$. Using this idea, we average squared dominant frequency vectors over small spatial neighborhoods. By halving the orientations of these "average squared" vectors and adding $\pi / 2$, we obtain the desired smooth estimates of the fingerprint local orientation field. Let $\nabla \varphi_{D}(x, y)=\left[U_{D}(x, y) V_{D}(x, y)\right]^{T}$ and consider a complex vector field $U_{D}(x, y)+j V_{D}(x, y)$. The squared field then has real part $U_{s, D}(x, y)=U_{D}^{2}(x, y)-V_{D}^{2}(x, y)$ and imaginary part $V_{s, D}(x, y)=2 U_{D}(x, y) V_{D}(x, y)$. These are spatially averaged over $3 w \times 3 w$ windows with center points $\left(x_{m} y_{n}\right)$ according to

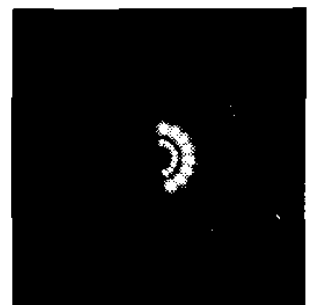

(a)

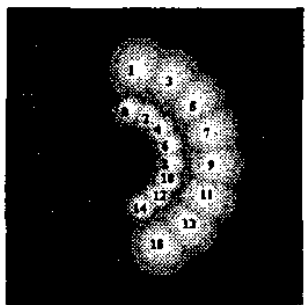

(b)
Fig. 1: (a) Frequency domaln representation of a 16-channel Gabor fiterbank for capturing the ridges and backgrounds of fingerprimt Images. (b) Detall view with filterbank channol numbers superimposed. Each filter has been independently scaled for display.

$$
\begin{aligned}
& J_{x}(m, n)=\sum_{p, q=-(3 w-1) / 2}^{(3 w-1) / 2} U_{s, D}\left(x_{m}+p, y_{n}+q\right) \\
& J_{y}(m, n)=\sum_{p, q=-(3 w-1) / 2}^{(3 w-1) / 2} V_{s, D}\left(x_{m}+p, y_{n}+q\right)
\end{aligned}
$$

The local ridge orientation field is then given by

$$
\theta(m, n)=\frac{1}{2} \tan ^{-1}\left(\frac{J_{y}(m, n)}{J_{x}(m, n)}\right)+\frac{\pi}{2} .
$$

The orientation field algorithm is illustrated in Fig. 3. The segmented fingerprint image from Fig. 2(d) appears again in Fig. 3(a). The orientations of the "average squared" frequency vectors (5),(6) are shown in Fig. 3(b), while the ridge orientation field (7) appears in Fig. 3(c).

\section{Reference Point Detection}

The proposed multi-resolution reference point detection algorithm is described in this section. The following steps are iteratively applied to the foreground of the segmented fingerprint image starting with $w=16$ :

1. Estimate the local orientation field $\theta(m, n)$ using window size $3 w \times 3 w$ as described in section 4 .

2. For each block pixel $\theta(m, n)$, compute the Poincare index $[1,11]$ along a $2 \times 2$ counterclockwise curve defined by $(m, n) \rightarrow(m+1, n) \rightarrow(m+1, n+1) \rightarrow(m, n+1)$ $\rightarrow(m, n)$.
3. Designate as reference points those block pixels $\theta(m, n)$ for which Poincaré $(i, j)=\pi$. For whorl and double loop type fingerprints, this will include both the concave and convex ridge core points.

4. In cases where two core points are detected, discriminate between the concave and convex ridge core points by summing the magnitudes of the local frequency orientation in the $3 \times 3$ neighborhood located above each core point (see Fig. 4(c); the cross mark is the location of the detected core point). The core point that gives the maximum sum is retained as the reference point.

Steps 1-4 are then applied iteratively two more times at finer resolutions $w=8$ and 4 , but only in the neighborhood of the reference point that was detected at the previous (coarser) scale.

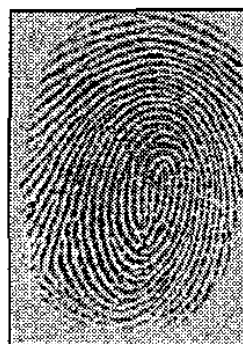

(a)

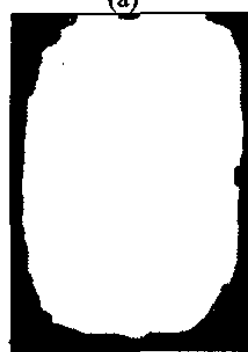

(c)

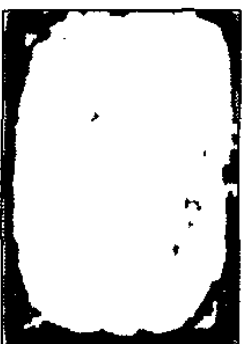

(b)

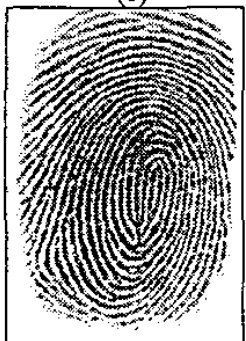

(d)
Figure 2: Segmentation example from FVC2000 DB2. (a) Original fingerprint. (b) Initial segmentation based on filterbank channel numbers. (c) Result after open-close and connected components labeling with minor region removal. (d) Final segmented fingerprint.

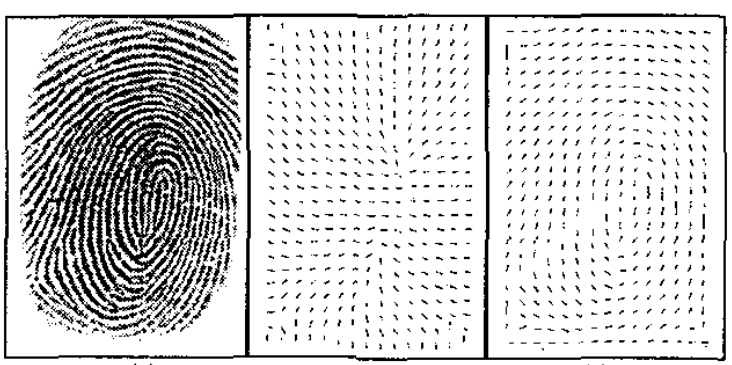

(a)

(b)

(c)

Fig. 3: Orlentation field estimation. (a) Segmented fingerprint image. (b) Orientation field computed for the "average squared" dominant frequency vectors. (c) Estimated ridge orientation field. 


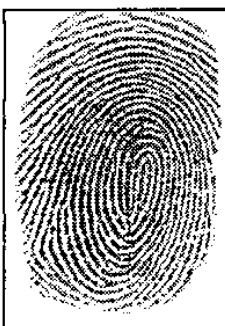

(a)

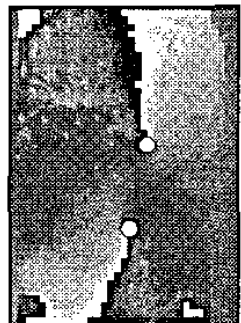

(b)

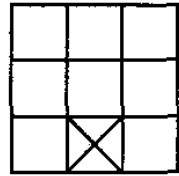

(c)
Fig. 4: Detecting the reference point. (a) Segmented fingerprint image. (b) Magnitude of the ridge orientation field with the detected corvex and concave core points marked by white circles. (c) The $3 \times 3$ region used for selecting the upper core point as the reference point.

\section{Experimental Results}

Our reference point detection algorithm was implemented and tested against the FVC 2000 Database 2 [7] and against a second database from the University of Bologna [8]. The filterbank design parameter $f$ described in Section 3 was calculated using estimated average inter-ridge distances for these two databases of 10 and 7 pixels, respectively. For comparison, we also implemented the reference point detection algorithm proposed recently in [1]. An unbiased graduate student was trained in the definition of reference point given in [1] and asked to manually identify the reference points of all 880 fingerprints in the FVC 2000 DB2 and all 168 fingerprints in the Bologna database from [8]. The arch type fingerprints were discarded from both databases because the core point is only poorly defined for fingerprints of the arch type; neither the algorithm of [1] nor our proposed algorithm can deliver consistent performance on arch type prints.

For each database, Table 1 gives the average Euclidean distance in pixels between the manually detected reference point and the reference points estimated by both the modulation domain algorithm proposed here and the algorithm given in [1]. For each algorithm, the standard deviation of the distance is also given. Typical experimental results for FVC $2000 \mathrm{DB} 2$ are given in Fig. 5. The arch type fingerprint in Fig. 5(e) and (j), while not included in calculation of the results reported in Table 1 , represents an interesting arch type case where both algorithms were able to identify a reference point.

\begin{tabular}{|c|c|c|}
\hline FVC 2000 DB2 & $\begin{array}{l}\text { Average distance from } \\
\text { manually detected } \\
\text { reference point (pixel) }\end{array}$ & $\begin{array}{l}\text { Standard } \\
\text { Deviation } \\
\text { ond }\end{array}$ \\
\hline Proposed algorithm & 16.35 & 22.30 \\
\hline Algorithm from [ $\mathrm{t}]$ & 26.45 & 29.91 \\
\hline Database from [8] & $\begin{array}{l}\text { Average distance from } \\
\text { manually detected } \\
\text { teference point (pixel) }\end{array}$ & $\begin{array}{l}\text { Standard } \\
\text { Deviation }\end{array}$ \\
\hline Proposed algorithm & 12.87 & 9.10 \\
\hline Algorithm from [1] & 45.04 & 48.34 \\
\hline
\end{tabular}

Table 1: Comparison summary.

\section{Conclusion}

We presented a new multi-resolution modulation domain algorithm for automatically detecting the reference point in fingerprint images. The mair novelty of the approach is that the Poincaré index is calculated over a ridge orientation field obtained from the frequency vectors in a computed dominant component AM-FM image rnodel rather than from the simple gradient field of the image intensities. As shown in Table1, for two well-known fingerprint databases the modulation domain algorithm achieves a tangible performance advantage over the technique proposed recently in [1].

\section{References}

[1] Anil K. Jain, Salil Prabltakar, Lin Hong, Sharath Pankanti, "Filterbank-Based Fingerprint Matching," IEEE Trans. Image Proc., vol. 9, no. 5, pp. 846-859, May 2000.

[2] M.S. Pattichis, G. Panayi, A.C. Bovik, Shun-Pin Hsu, "Fingerprint Classification using an AM-FM model", IEEE Trans. Image Proc., vol. 10, no. 6, pp. 951-954, Jun. 2001.

[3] J.P. Havlicek, D.S. Harding, and A.C. Bovik, "Multidimensional quasi-eigenfunction approximations and multicomponent AM-FM models," IEEE Trans. Image Proc., vol. 9, no. 2, pp. 227-242, Feb. 2000.

[4] J.P. Havlicek, A.C. Bovik, and D. Chen, "AM-FM Image Modeling and Gabor Analysis," in Visual Information Representation, Communication, and Image Processing, C.W. Chen and Y. Zhang, eds., Marcel Dekker, New York, 1999, pp. 343-385.

[5] S.T. Acton, D. Prasad Mukherjee, J.P. Havlicek, A.C. Bovik, "Oriented Texture Completion by AM-FM reactiondiffusion", IEEE Trans. Image Proc., vol. 10, no. 6, pp. 885-896, Jun. 2001.

[6] J.P. Havlicek, J.W. Havlicek, and A.C. Bovik, "The analytic image," in Proc. IEEE Int. Conf. Image Processing, Santa Barbara, CA, Oct. 26-29, 1997, pp.446449.

[7] D. Maio, D. Maltoni, R. Cappelli, J.L. Wayman, and A.K. Jain, "FVC2000: Fingerprint Verification Competition," Biolab intemal report, Univ. of Bologna, Italy, Sept. 2000, available at http://bias.csr.unibo.it/fvc2000/.

[8] Biometrics Systems Lab., University of Bologna, CesenaItaly, http://www.csr.unibo.it/research/biolab/bio_tree.html.

[9] Kass M. and Witkin A., "Analyzing Oriented Patterns", Computer Vision, Graphics, and Image Processing, vol. 37, pp. 362-385, 1987.

[10] Rao A.R., A Taxonomy for Texture Description and Identification, Springer-Verlag, New York, 1990.

[11] M. Kawagoe and A. Tojo, "Fingerprint pattern classification", Pattern Recognition, vol. 17, pp. 295-303, 1984. 

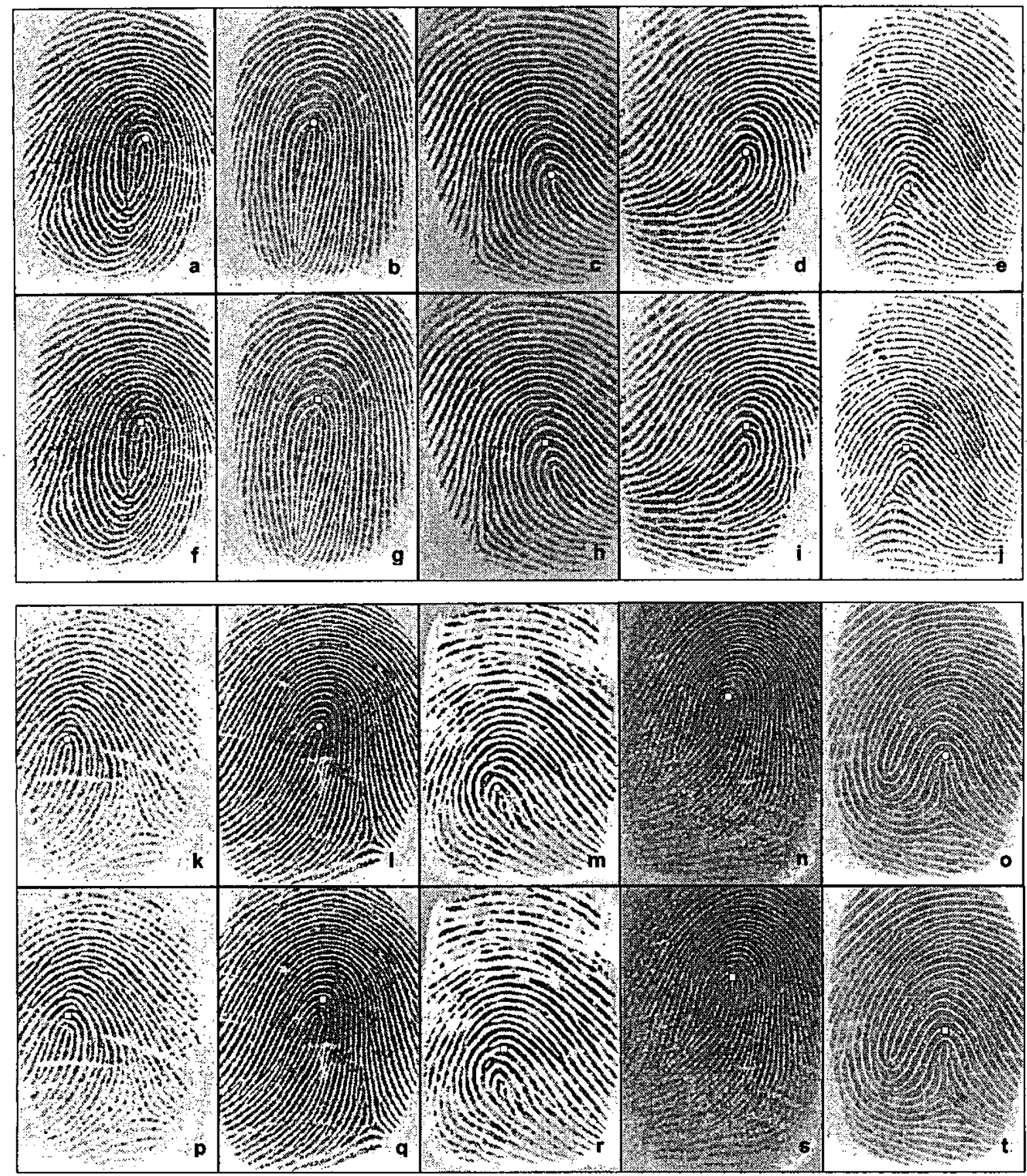

Fig. 5: Results from FVC 2000 Database 2. (a)-(e), (k)-0): proposed algorithm (estimated reference point shown as a white circle). (f)-(l), (p)-(t): corresponding results from the algorithm given in [1] (reference point estimate shown as a white square). 